\title{
Beyond syntax: Language-related positivities reflect the revision of hierarchies
}

\author{
Ina Bornkessel, ${ }^{\text {CA }}$ Matthias Schlesewsky' and Angela D. Friederici
}

Max-Planck-Institute of Cognitive Neuroscience, P.O. Box 5003 55, 04303 Leipzig; ' Department of Linguistics, University of Potsdam, Germany

\author{
${ }^{\mathrm{CA}}$ Corresponding Author
}

Received I 9 December 200I; accepted 3 January 2002

\begin{abstract}
On the basis of an experiment using event-related brain potentials (EPRs), we argue that a characterisation of language-related positivities as necessarily syntax-related is too restrictive. Our data show that, in verb-final German clauses, the processing of a verb which disconfirms the expectations with regard to the hierarchical thematic structure of a sentence (who is doing what to whom)

Key words: Early positivity; ERPs; Sentence processing; Thematic reanalysis
\end{abstract}

gives rise to an early $(200-600 \mathrm{~ms})$ parietal positivity. Thus, positive ERP components elicited during language processing appear to be related to operations (most often revisions) applying to hierarchically structured linguistic information in general, rather than to syntactic structure in particular. NeuroReport 13:36 |-364 (c) 2002 Lippincott Williams \& Wilkins.

\section{INTRODUCTION}

Since Kutas and Hillyard's seminal work on the application of the event-related brain potentials (ERPs) methodology to language processing [1], numerous studies concerned with how humans understand language have made use of this method. In this way, a number of language-related ERP components have been identified, among them the P600 or syntactic positive shift, a parietally distributed positivity between $\sim 500$ and $900 \mathrm{~ms}$ after the onset of a critical word. This component has been associated with the processing of syntactic information in general [2], with processes of syntactic reanalysis and repair [3-5], and with syntactic integration cost [6]. An early positive component (P345) interpreted as reflecting the diagnosis preceding reanalysis has also been reported [7,8]. Thus, language-related positivities have hitherto been generally associated with a broad range of syntax-related operations.

The present study examines the hypothesis that languagerelated positivities may be elicited by operations (e.g. reanalyses) applying to hierarchical information in general, rather than to syntactic information per se. To this end, we draw upon a type of information that is not syntactic but nevertheless hierarchically ordered, namely thematic information.

Thematic information essentially determines who is doing what to whom in a given sentence. In the sentence John kissed Mary, for example, John is the initiator of the kissing event (the Agent) and Mary the undergoer of this event (the Patient). Thus, thematic information provides a general conceptual specification of the relations between the arguments of a sentence to one another and to the verb. Most importantly for present purposes, thematic information is hierarchically structured by way of thematic dependencies. Broadly speaking, this type of dependency arises since if one participant of a predicate is causally affected, the predicate necessarily selects a causer as another participant' $[9$, p. 52].

In the following, we will only be concerned with this hierarchical nature of the thematic relations between the arguments (i.e. it will be irrelevant whether an argument represents a willfully causing Agent or a, perhaps accidental, Causer, for example). In this way, we may refer to the thematically higher argument as a Proto-Agent and to the thematically lower argument as a Proto-Patient $[9,10]$.

Previous psycholinguistic studies have shown that languages with morphological case marking allow an immediate mapping of arguments to thematic (proto-)roles, which is made without reference to the verb [11]. This is possible because a particular case marking is only compatible with a certain subset of thematically relevant features (e.g. control), thus restricting the potential range of proto-roles that this argument may be mapped onto. The immediate association of arguments to proto-roles implies that, in verb-final clauses such as the German (1), a hierarchical thematic ordering is established between the arguments on the basis of their case marking even before the verb is encountered. Thus, the nominative argument der Professor ('The professor') will be assigned the Proto-Agent role and the dative argument dem Gärtner ('the gardener') the Proto-Patient role.

(1)...dass der Professor dem Gärtner dankt/gefällt

...that [the professor]-a NOMINATIVE[the gardener]- DATIVE thanks/pleases

...that the Professor thanks/is pleasing to the gardener

This preferential thematic ordering is confirmed by a clausefinal active (Agent-Patient) verb such as danken ('to thank'), 
but disconfirmed by an object-experiencer verb such as gefallen ('to be pleasing to'), since this type of verb assigns the higher-ranked experiencer role to the dative object $[9,12]$. Thus, when a verb-final sentence is completed by an object-experiencer verb, the hierarchical thematic ordering between subject and object must be revised. If the hypothesis that language-related positivities are a reflex of hierarchically ordered information rather than syntactic information is correct, object-experiencer verbs in sentences such as (1) should give rise to a positivity in comparison to their active counterparts.

In order to ensure that any effects observed for objectexperiencer verbs are indeed attributable to a thematic reanalysis, we must contrast constructions such as (1) with constructions in which no thematic preferences arise before the verb is processed. Since such preferences are crucially linked to the morphological case marking of an argument, sentences analogous to (1) but without unambiguous case marking should be suitable controls in this regard (cf. 2).

\section{(2)...dass Maria Sängerinnen dankt/gefällt}

...that Maria- ${ }_{\text {AMB }}$ singers- ${ }_{\text {AMB }}$ thanks/pleases ...that Maria thanks/is pleasing to singers

In (2), both arguments are three-way ambiguous between nominative, accusative, and dative, i.e. maximally underspecified with regard to thematic features. In view of this unrestrictedness, no hierarchical thematic ordering can be established before the verb is reached and therefore no effect of verb type should be observable.

\section{MATERIALS AND METHODS}

Twenty students of the University of Leipzig participated in the experiment (11 female; age 20-27 years; mean 22.6 years) after giving informed consent. All were right handed, native speakers of German with normal or corrected-to-normal vision. The participants were paid DM13/h.

The experimental sentences comprised a matrix clause of the form Maria hörte ('Maria heard') and an embedded clause of the form shown in $(1 / 2)$. Each sentence was completed by an adjunct clause in order to avoid placing the crucial verb clause-finally. The experimental design involved a manipulation of the factors verb type (VERB: active vs object-experiencer) and ambiguity (AMB: unambiguous vs ambiguous case marking). Participants were presented with 80 sentences for each of the four critical conditions in a randomised manner. Each condition included an equal number of subject- and object-initial sentences in order to control for possible influences of word order. After each experimental sentence, participants were required to judge whether a subsequently presented declarative sentence correctly expressed the content of the preceding sentence or not. This comprehension task required the answer 'yes' equally as often as the answer 'no', and the incorrect sentences involved an inverted assignment of grammatical functions (subject and object) to the arguments.

Sentences were presented visually in the centre of a computer screen in a phrase-by-phrase manner (i.e. NPs were presented as a whole). Single words were presented for $450 \mathrm{~ms}$ and phrases for $500 \mathrm{~ms}$ with an inter-stimulus interval of $100 \mathrm{~ms}$. Participants were asked to avoid move- ments and to only blink their eyes between their response to the comprehension task and the presentation of the next sentence. The experimental session began with a short training session followed by 8 experimental blocks comprising 40 sentences each, between which the participants took short breaks. The entire experiment (including electrode preparation) lasted $\sim 2.5 \mathrm{~h}$.

The EEG was recorded by means of $58 \mathrm{Ag} / \mathrm{AgCl}$ electrodes fixed at the scalp by means of an elastic cap (Electro Cap International). The ground electrode was positioned above the sternum. Recordings were referenced to the left mastoid, but rereferenced to linked mastoids offline. The electro-oculogram (EOG) was monitored by means of electrodes placed at the outer canthus of each eye for the horizontal EOG and above and below the participant's right eye for the vertical EOG. Electrode impedances were kept $<5 \mathrm{kOhm}$.

All EEG and EOG channels were amplified using a Neuroscan synamps amplifier (DC to $50 \mathrm{~Hz}$ ) and recorded continuously with a digitisation rate of $250 \mathrm{~Hz}$. The plots of grand average ERPs were smoothed off-line with a $10 \mathrm{~Hz}$ low pass filter, but all statistical analyses were computed on unfiltered data.

Average ERPs were calculated per condition per participant from the onset of the critical stimulus item (i.e. the verb) to $1000 \mathrm{~ms}$ post-onset, before grand averages were computed over all participants. Averaging took place relative to a baseline interval from -200 to $0 \mathrm{~ms}$ before the onset of the verb. Trials for which the comprehension task was not performed correctly were excluded from the averaging procedure, as were trials containing ocular, amplifier saturation or other artefacts (the EOG rejection criterion was $40 \mu \mathrm{V}$ ).

For the statistical analysis of the ERP data, repeated measures ANOVAS were calculated for mean amplitude values per time window per condition. The statistical analysis was carried out in a hierarchical manner, i.e. only significant interactions $(p<0.05)$ were resolved. Additionally, no main effects of or interactions between topographical factors will be reported.

Topographical factors were chosen as follows. For the midline electrodes, the factor Electrode included the eight electrodes AFZ, FZ, FCZ, CZ, CPZ, PZ, POZ and OZ as levels; for the lateral electrodes, the factors Hemisphere (left vs right) and Region (anterior, posterior) were fully crossed, thus giving rise to the following four lateral regions of interest (ROIs): left-anterior (AF7, AF3, F7, F5, F3, FT7, FC5, FC3), left-posterior (TP7, CP5, CP3, P7, P5, P3, PO7, PO3), right-anterior (AF8, AF4, F8, F6, F4, FT8, FC6, FC4), rightposterior (TP8, CP6, CP4, P8, P6, P4, PO8, PO4).

\section{RESULTS}

Figure 1 and Fig. 2 show grand average ERPs at the position of the verb for unambiguous and ambiguous sentences, respectively. As is apparent from Fig. 1, for unambiguous structures, object-experiencer verbs give rise to a posterior positivity between 200 and $600 \mathrm{~ms}$ in comparison to active verbs. By contrast, no such difference is apparent for the ambiguous structures (Fig. 2).

For the time-window $200-600 \mathrm{~ms}$, the statistical analysis for the lateral electrodes revealed a main effect of 

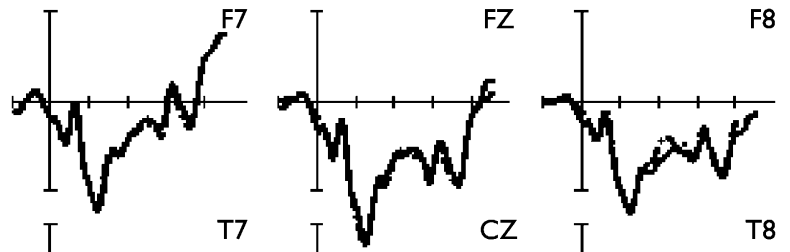

F8
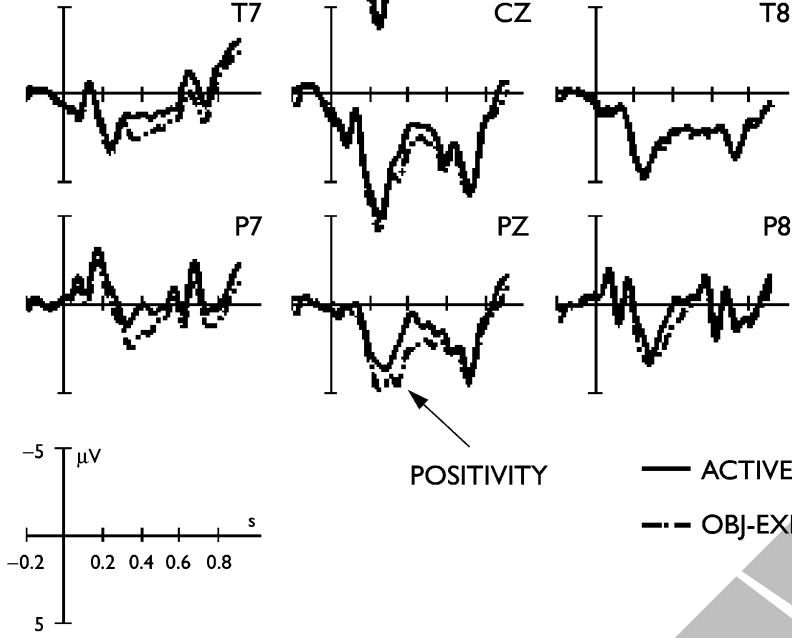

POSITIVITY
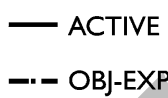

Fig. I. Grand average ERP elicited by active vs object-experiencer verbs (onset at the vertical line) for unambiguous sentences. Negativity is plotted upwards.
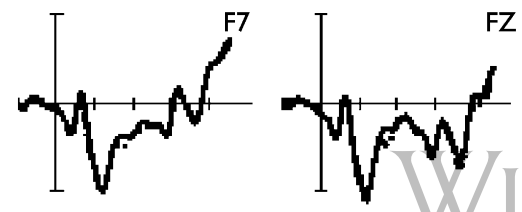

FZ
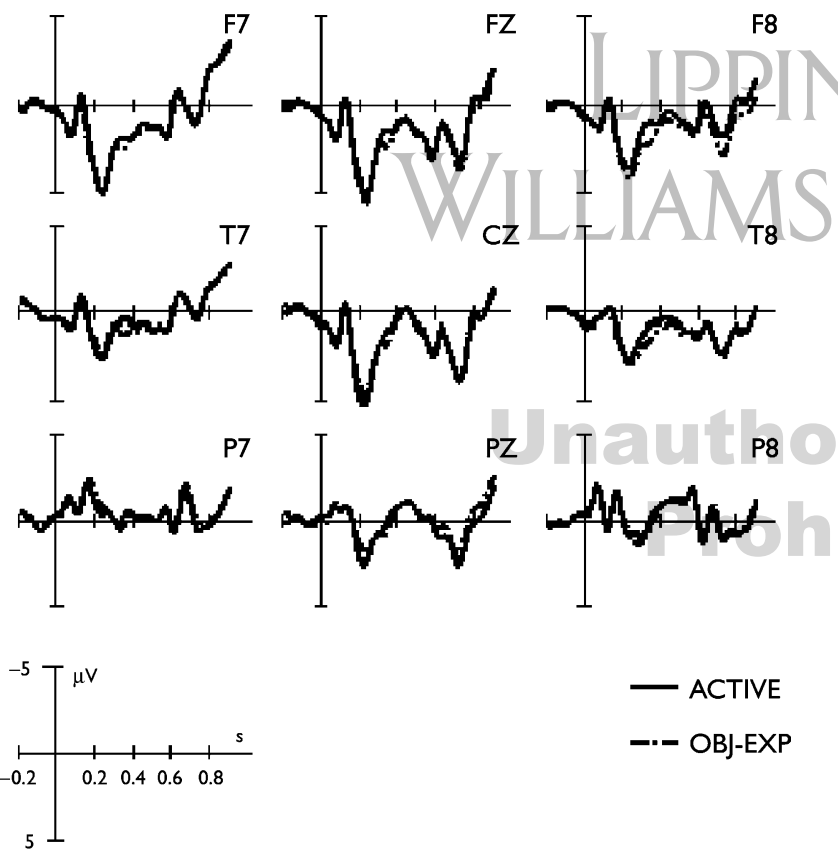

$-\cdot-\mathrm{OBJ}-\mathrm{EXP}$

Fig. 2. Grand average ERPs elicited by active vs object-experiencer verbs (onset at the vertical line) for ambiguous sentences. Negativity is plotted upwards.

AMB $(F(1,19)=21.02, p<0.001)$, which was due to more negative waveforms for ambiguous in comparison to unambiguous structures. Furthermore, there were significant interactions for Hemisphere $\times$ AMB $(F(1,19)=5.11$, $p<0.04)$ and Region $\times$ AMB $\times \operatorname{VERB~} \quad(\mathrm{F}(1,19)=7.15$, $p<0.02)$. Planned comparisons for each of the four ROIs revealed significant main effects of $\mathrm{AMB}$ for the right-anterior $\quad(F(1,19)=11.64, p<0.01)$, right-posterior $(\mathrm{F}(1,19)=34.84, \quad p=0.0001)$ and left-posterior quadrants $(\mathrm{F}(1,19)=8.54, p<0.01)$, as well as a marginal main effect of $\mathrm{AMB}$ in the left-anterior quadrant $(\mathrm{F}(1,19)=3.94$, $p<0.07)$. The interaction AMB $\times$ VERB was significant in the left-posterior $(\mathrm{F}(1,19)=9.78, p<0.01)$ and marginal in the right-posterior quadrant $(\mathrm{F}(1,19)=3.71, p<0.07)$. Resolving the left-posterior interaction $A M B \times V E R B$ by $A M B$ revealed that only unambiguous structures gave rise to a VERB effect $(\mathrm{F}(1,19)=19.05, p<0.001)$, with object-experiencer verbs more positive than active verbs. There was no such effect for the ambiguous structures $(\mathrm{F}<1)$.

With regard to the midline electrodes, there was again a significant main effect of AMB $(\mathrm{F}(1,19)=23.81, p=0.0001)$, with ambiguous structures more negative than unambiguous structures, as well as an interaction $\mathrm{AMB} \times \mathrm{VERB}$ $(\mathrm{F}(1,19)=6.76, p<0.02)$. Planned comparisons for unambiguous and ambiguous sentences revealed an effect of VERB for unambiguous $(\mathrm{F}(1,19)=7.71, p<0.02$, object-experiencers more positive), but not for ambiguous structures $(\mathrm{F}<1)$.

\section{DISCUSSION}

Our data show that in unambiguously case marked German verb-final clauses, object-experiencer verbs give rise to an early parietal positivity in contrast to active verbs. There was no such difference in analogous structures with ambiguous case marking.

Crucially, the early positivity was observable independently of syntactic manipulations. Furthermore, this effect cannot be ascribed to a simple lexical difference between active and object-experiencer verbs, since no main effect of verb class was observable for ambiguous structures. Rather, these results support the assumption that the non-confirmation of a preferential thematic ordering between arguments leads to a thematic reanalysis. The hierarchical reordering of non-syntactic information thus required is reflected in a positive deflection in the ERP. When no thematic hierarchising can take place before the verb is processed, as is the case with ambiguous structures which provide no specification of thematic features, the processing of object-experiencer verbs does not differ from that of active verbs.

The finding that revisions of non-syntactic, but nevertheless hierarchical information lead to a positivity in the ERP indicates that a characterisation of language-related positivities in terms of syntax-related operations is too restrictive. It rather appears more accurate to describe early and late positivities as reflecting operations related to the manipulation of hierarchical structure in general, of which syntactic structure is, of course, a special case.

Why, then, should the latency of language-related positivities vary? One possibility is that the latency differences reflect which processing stage the operations (e.g. reanalysis) giving rise to the positivity are associated with. Thus, Friederici's neurocognitive model of sentence processing $[5,13]$ assumes three processing phases, the first involving word category processing and the second being constituted by parallel, but independent functional and 
interpretative processes resulting from the application of the lexical information associated with the word currently being processed. In the third stage, reanalysis/repair processes (reflected by the P600) set in, when the syntactic and conceptual representations built up in the second phase cannot be mapped onto one another. Thus, the P600 is a late positivity because it can only set in after phase 2 of processing is complete. In the present experiment, however, reanalysis could take place solely within the interpretative processing route of phase 2 , thus giving rise to an early positivity.

\section{CONCLUSION}

Language-related positivities reflect processing operations applying to hierarchically structured information, of which syntactic information is but a special case. The latency of these components appears to be influenced by the types of information upon which the respective processes operate.

\section{REFERENCES}

1. Kutas M and Hillyard SA. Science 207, 203-205 (1980).

2. Hagoort P, Brown C and Groothusen J. Lang Cogn Processes 8, 43-83 (1993).

3. Osterhout L, Holcomb PJ and Swinney DA. J Exp Psychol Learn Mem Cogn 20, 786-803 (1994).

4. Osterhout L and Holcomb PJ. J Mem Lang 31, 785-804 (1992).

5. Friederici AD. Brain Lang 50, 259-281 (1995).

6. Kaan E, Harris A, Gibson E et al. Lang Cogn Processes 15, 159-201 (2000).

7. Mecklinger A, Schriefers H, Steinhauer K et al. Mem Cogn 23, 477-494 (1995).

8. Friederici AD. Diagnosis and reanalysis: two processing aspects the brain may differentiate. In: Fodor JD and Ferreira F, eds. Reanalysis in Sentence Processing. Dordrecht: Kluwer; 1998, pp. 177-200.

9. Primus B. Cases and thematic roles. Tübingen: Niemeyer; 1999.

10. Dowty D. Language 67, 547-619 (1991).

11. Frisch S and Schlesewsky M. Neuroreport 12, 3391-3394 (2001).

12. Wunderlich D. Linguistic Inquiry 28, 27-68 (1997).

13. Friederici AD. The Neurobiology of Language Comprehension. In: Friederici $\mathrm{AD}$, ed. Language Comprehension: A Biological Perspective. Berlin: Springer; 1999, pp. 265-292.

\section{Acknowledgements: The research reported here was supported by the grants FOR 375/I- 4 awarded to M.S. and A.F. and FI 848/I-I awarded to A.F. from the Deutsche Forschungsgemeinschaft (DFG). We would like to thank Stefan Frisch and Gisbert Fanselow for helpful comments and discussions and Ulrike Barth for the data acquisition.}
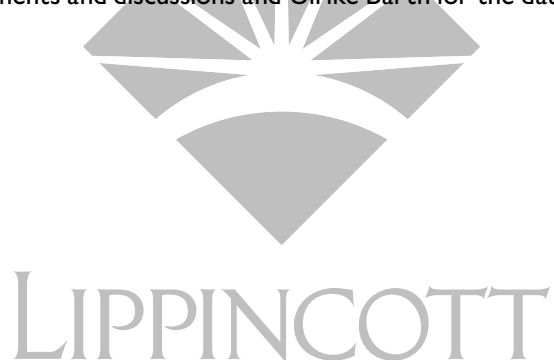